05

\title{
Безрезонаторная генерация на ионах молекулярного азота в воздушной лазерной плазме
}

\author{
(С) И.А. Зятиков, В.Ф. Лосев \\ Институт сильноточной электроники Сибирского отделения РАН, \\ 634055 Томск, Россия \\ e-mail: kidnapper66@gmail.com
}

Поступила в редакцию 20.12.2021 г.

В окончательной редакции 20.12.2021 г.

Принята к публикации 30.12.2021 г.

Исследованы временные и спектральные характеристики генерации на переходе $B^{2} \Sigma_{u}^{+}-X^{2} \Sigma_{g}^{+}$ионов молекулярного азота в лазерной плазме, создаваемой импульсом излучения с центральной длиной волны $950 \mathrm{~nm}$, длительностью $60 \mathrm{fs}$ и энергией $10 \mathrm{~mJ}$. Анализируются параметры активной среды и обсуждается возможный механизм формирования пикосекундной длительности импульса излучения.

Ключевые слова: генерация, лазерная плазма, молекулярные ионы азота, временной профиль импульса, спектр.

DOI: $10.21883 /$ OS.2022.04.52264.63-21

\section{Введение}

В последние годы большой интерес проявляется к генерации излучения на ионах молекулярного азота на длинах волн 391.4 и $427.8 \mathrm{~nm}$, которая возникает в лазерной плазме, создаваемой интенсивным фемтосекундным лазерным импульсом [1-5]. Данные две линии соответствуют переходу первой отрицательной системы иона молекулярного азота $B^{2} \Sigma_{u}^{+}\left(v^{\prime}=0\right)-X^{2} \Sigma_{g}^{+}$ $(v=0,1)$, где $v^{\prime}$ и $v$ - колебательные квантовые числа верхнего и нижнего электронных уровней. Интерес к такому источнику когерентного излучения в УФ области спектра обусловлен возможностью применения в различных областях спектроскопии и медицины, а также для создания лазерной плазмы (активной среды) в воздухе на расстоянии в несколько километров от лазера накачки, что очень выгодно для дистанционного зондирования атмосферы [6]. Под генерацией подразумевается высоконаправленное когерентное вынужденное излучение в активной среде на ионах молекулярного азота без резонатора. Данное излучение было впервые обнаружено путём накачки лазерным импульсом в средней ИК области [1]. Позже оно наблюдалось при накачке импульсом на других длинах волн, включая $1.03 \mu \mathrm{m}$ [5], $950 \mathrm{~nm}$ [7,8], $800 \mathrm{~nm}$ [2-4]. Было показано, что для возникновения генерации необходимо использовать внешнее дополнительное (затравочное) излучение на длине волны соответствующего перехода иона азота, в качестве которого выступает излучение гармоник накачки или суперконтинуума (СК). В более поздних работах генерация была получена как в присутствии внешнего затравочного излучения [4], так и без внешней затравки [2,3]. В последнем случае считается, что в качестве запускающего импульса выступают фотоны СК на рабочей длине волны, которые возникают в лазерной плазме во время импульса накачки. При этом, как правило, генерацию получают только на одной спектральной линии даже в присутствии затравочного излучения от CK.

В связи со сложными процессами возникновения генерации до настоящего времени всё ещё не определён физический механизм, лежащий в основе её возникновения, и в литературе продолжается обсуждение данного вопроса $[9,10]$. Для того, чтобы определить природу возникновения генерации, необходимо знать все её характеристики и их зависимости от условий эксперимента. Одной из важных характеристик генерации является длительность импульса. Основным методом для измерения длительности импульса является кросскорреляционный метод [11-13]. В данном методе записывается сигнал суммарной частоты как функция задержки между приходом импульса генерации и накачки при их частотном смешивании в нелинейном кристалле. В этом случае известно, что процесс частотного смешивания является нелинейным. В результате временной профиль импульса генерации будет отличаться от действительного. Реальный временной профиль генерации может быть получен с помощью прямых измерений стрик-камерой с высоким временным разрешением.

В первых работах предполагалось, что длительность импульса генерации является спектрально ограниченной. Поскольку типичная спектральная ширина на полувысоте генерации составляет $\sim 0.28$ и $\sim 0.24 \mathrm{~nm}$ для длин волн 427.8 и $391.4 \mathrm{~nm}$, то была оценена спектрально ограниченная длительность в 0.96 и 0.93 ps соответственно [3]. Однако дальнейшие эксперименты по измерению длительности кросс-корреляционным методом и стрик-камерой с разрешением 0.74 [7] и 8 ps [14] показали, что длительность импульса генерации может быть больше спектрально ограниченной (1.15-7.8 ps) [7,9,14- 


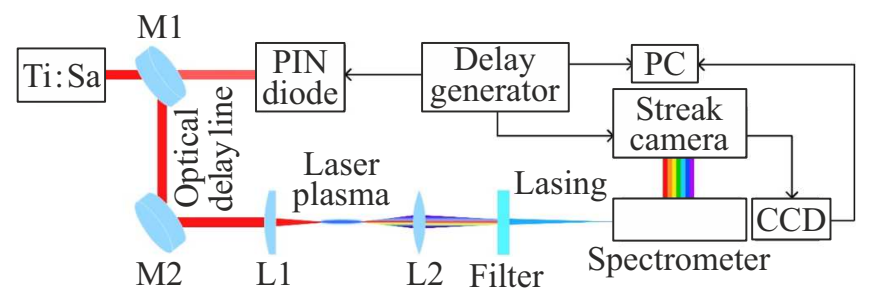

Рис. 1. Экспериментальная схема: лазерная система Ti:Sa, поворотные зеркала, фокусирующая (L1) и собирающая (L2) линзы, лазерная плазма, световой фильтр и регистрирующие элементы.

16], а её величина зависит от экспериментальных условий, которые отличаются друг от друга в различных работах. Также принято считать, что фемтосекундный запускающий импульс усиливается по мере прохождения по активной среде, но на выходе регистрируется пикосекундный импульс. Механизм удлинения и причины, от которых зависит длительность импульса генерации, до настоящего времени в литературе не обсуждались.

В настоящей работе приводятся результаты исследования временных и спектральных характеристик импульса генерации на ионах молекулярного азота в воздухе для различных параметров лазерной плазмы. Анализируются параметры активной среды и обсуждается возможный механизм формирования пикосекундной длительности импульса излучения.

\section{Экспериментальная аппаратура и методики измерения}

Эксперименты проводились на лазерной системе $\mathrm{Ti}: \mathrm{Sa}$, которая формировала спектрально ограниченный импульс на центральной длине волны $950 \mathrm{~nm}$. Длительность импульса накачки составляла $60 \mathrm{fs}$ на полувысоте амплитуды, энергия - $10 \mathrm{~mJ}$ и диаметр $1.5 \mathrm{~cm}$ (уровень интенсивности $\left.1 / e^{2}\right)$. Частота следования импульсов $10 \mathrm{~Hz}$ [7]. Пучок накачки фокусировался плосковыпуклой линзой, фокусное расстояние которой варьировалось от 15 до $50 \mathrm{~cm}$, для создания лазерной плазмы и получения генерации в воздухе. Экспериментальная схема представлена на рис. 1 .

Размеры лазерной плазмы оценивались из фотографий цифровой камерой сбоку плазмы. Временные измерения генерации проводились на стрик-камере (Hamamatsu Universal Streak Camera C10910). Стрик-камера имеет две развёртки: быструю (минимальная развёртка 100 ps) и медленную (минимальная развёртка $1 \mathrm{~ns}$ ). Максимальное временное разрешение стрик-камеры составляет 0.644 ps. Эксперименты проводились на быстрой развёртке с шириной щели хронографа $20 \mu \mathrm{m}$. По сигналу c pin-диода камера запускалась на частоте следования импульсов накачки. Для компенсации времени срабатывания блока развёртки стрик-камеры излучение проходило настраиваемую оптическую линию задержки и затем фокусировалось линзой для создания лазерной плазмы. Длина оптической линии задержки составляла $11 \mathrm{~m}$. При этом параметры генерации не изменялись при создании лазерной плазмы вблизи $(\sim 3 \mathrm{~m})$ и на расстоянии $11 \mathrm{~m}$ от источника накачки, а профиль лазерного пучка практически не изменялся после прохождения данного пути. Энергия генерации на ионах азота была измерена на измерителе энергии лазерных импульсов OPHIR 3A-ROHS. Спектральные измерения генерации проводились на спектрометре Acton SpectraPro SP-2300, который был совмещён со стрик-камерой (разрешение $0.23 \mathrm{~nm}$ ), а также использовался широкополосный Ocean Optics HR-4000CG-UV-NIR с разрешением $0.75 \mathrm{~nm}$. Для измерений, близких к разрешающей способности прибоpa $B$, реальное значение $C$ оценивалось по следующей формуле:

$$
C=\sqrt{\left(A^{2}-B^{2}\right)}
$$

где $A$ - измеренная длительность импульса или спектральная ширина.

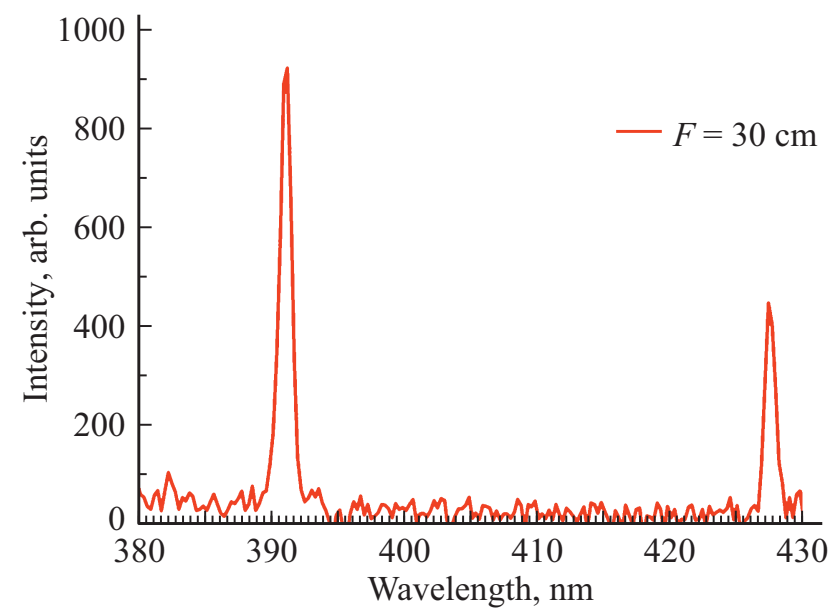

Рис. 2. Спектр генерации на длинах волн 391.4 и $427.8 \mathrm{~nm}$ в воздухе, записанный на спектрометре Ocean Optics HR4000CG-UV-NIR.

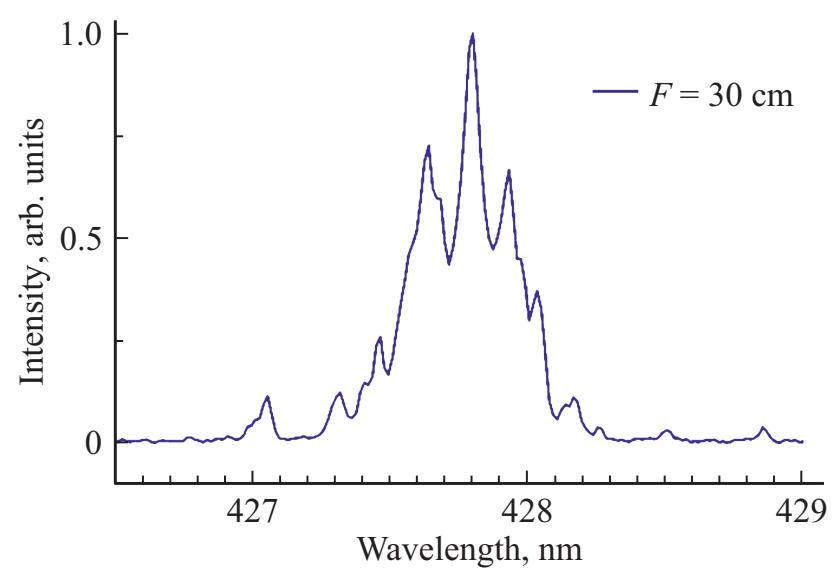

Рис. 3. Нормированный спектр генерации на длине волны $427.8 \mathrm{~nm}$ для $F=30 \mathrm{~cm}$. 


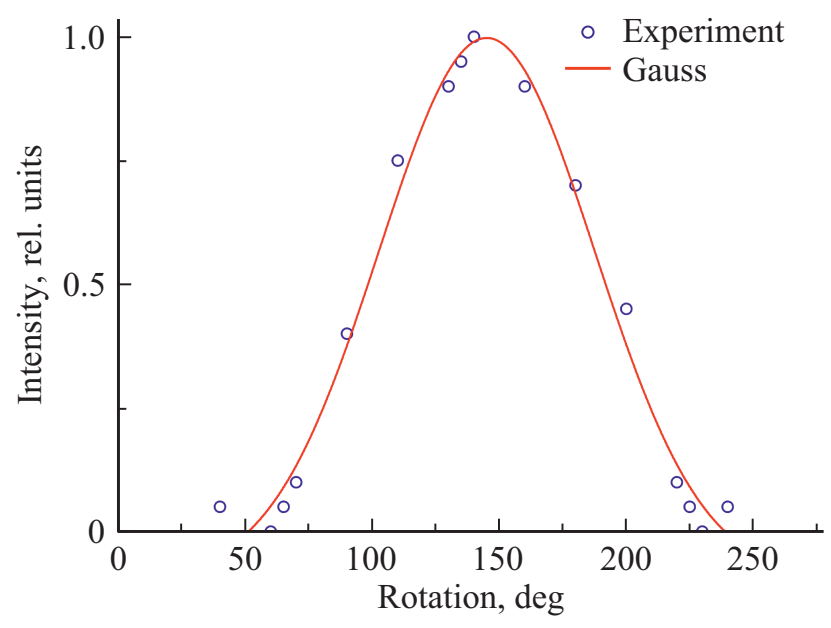

Рис. 4. Поляризация излучения на длине волны $391.4 \mathrm{~nm}$.

\section{Результаты и обсуждение}

\section{Исследование спектра генерации и поляризации излучения на молекулярных ионах азота в лазерной плазме}

В наших экспериментах были исследованы условия, при которых возможно получать одновременно две линии генерации на длинах волн 391.4 (0-0) и $427.8 \mathrm{~nm}$ (0-1), либо только одну из них. Ранее в большинстве экспериментов, проведённых нами при атмосферном давлении, наблюдалась только одна линия $427.8 \mathrm{~nm}$. Однако позднее при фокусировке излучения накачки с энергией $10 \mathrm{~mJ}$ линзой $\mathrm{c}$ фокусным расстоянием $30 \mathrm{~cm}$ была получена генерация на двух длинах волн одновременно (рис. 2). При этом обнаружено, что при внесении астигматизма за счёт небольшого наклона фокусирующей линзы изменялась интенсивность линий генерации вплоть до исчезновения одной из них.

Прежде уже была продемонстрирована возможность одновременного получения двух линий генерации на молекулярных ионах азота в воздухе [15]. Однако в большинстве случаев в режиме без внешнего затравочного сигнала в воздухе или в азотной камере с давлением до $750 \mathrm{mbar}$ получают генерацию только на длине волны $427.8 \mathrm{~nm}[3,11]$. Вторую линию на длине волны $391.4 \mathrm{~nm}$ получают, как правило, в режиме с внешним затравочным сигналом либо без него в азотной камере с низким давлением (до 200 mbar) [12, 17].

Измерения ширины спектра генерации проводились на спектрометре Acton SpectraPro SP-2300. Типичная ширина спектра на длине волны $427.8 \mathrm{~nm}$ представлена на рис. 3. Фокусировка осуществлялась линзой с $F=30 \mathrm{~cm}$.

С учётом формулы (1) реальная ширина спектра составляет $0.29 \mathrm{~nm}$. Изменение удельной мощности накачки не приводило к значительному изменению ширины спектра. Полученное значение ширины спектра находится в хорошем согласии с литературными данными в похожих экспериментальных условиях $[3,11]$. Колебания на спектре генерации, вероятно, связаны с вращательными уровнями $\mathrm{N}_{2}^{+}$, которые также наблюдались и в других работах $[4,11,17]$.

Исследование поляризации полученного излучения показало, что она является линейной и совпадает с поляризацией накачки и СК (рис. 4). Кроме этого, мощность импульса генерации была $\left(\sim 10^{6} \mathrm{~W}\right)$ существенно выше, чем у спонтанного излучения, которое мы не могли зарегистрировать. Это, как и в других работах, является доказательством вынужденного характера излучения на ионах молекулярного азота.

\section{Исследование формы импульса генерации}

Исследование временных параметров импульса генерации проводилось на стрик-камере в воздухе при различных значениях удельной мощности накачки. Варьирование мощности накачки обеспечивалось изменением фокусного расстояния линзы. Другие параметры излучения накачки (энергия - $10 \mathrm{~mJ}$, длительность - $60 \mathrm{fs}$, длина волны - $950 \mathrm{~nm}$ ) оставались фиксированными. На рис. 5 представлены измеренные формы импульса генерации на длине волны $427.8 \mathrm{~nm}$ при различных значениях фокусного расстояния линзы. С учётом формулы (1) реальные длительности импульса на полувысоте амплитуды составляют $1.15,3.7$ и 4.6 ps при фокусировке линзой с $F=15,30$ и $50 \mathrm{~cm}$ соответственно. Полученные значения длительности импульса находятся в хорошем согласии с литературными данными в похожих экспериментальных условиях, но измеренными косвенным кросс-корреляционным методом [12, 15]. Стоит отметить, что в наших условиях длительность импульса генерации увеличивается с увеличением фокусного расстояния линзы и уменьшением удельной мощности накачки.

На рис. 6 представлена измеренная длительность импульса генерации на длине волны $391.4 \mathrm{~nm}$ в воздухе при фокусном расстоянии линзы $F=30 \mathrm{~cm}$. Согласно формуле (1), реальная длительность импульса на полувысоте амплитуды для $391.4 \mathrm{~nm}$ составляет $2.1 \mathrm{ps}$. Периодические модуляции амплитуды во временных профилях 391.4 и $427.8 \mathrm{~nm}$ с интервалом в $\sim 2$ ps соответствуют полупериоду вращения $\mathrm{N}_{2}^{+}\left(B^{2} \Sigma_{u}^{+}\right)[18]$.

Стоит отметить, что в наших исследованиях длительности импульса генерации на длинах волн 391.4 и $427.8 \mathrm{~nm}$, измеренные в одних экспериментальных условиях, отличаются друг от друга в 1.68 раз. По литературным данным в одних экспериментальных условиях длительность импульса генерации на длине волны $427.8 \mathrm{~nm}$ была примерно в 2 раза больше, чем на $391.4 \mathrm{~nm}[11,12]$, что достаточно хорошо коррелирует с нашими результатами. Данное явление можно объяснить, если привлечь релаксацию с колебательного уровня $X(1)$ на $X(0)$. При одновременных переходах $B(0)-X(1)$ (длина волны $427.8 \mathrm{~nm}$ ) и $B(0)-X(0)$ (длина волны $391.4 \mathrm{~nm}$ ) эта релаксация приведёт к дополнительному расселению уровня $X(1)$ и, наоборот, к засе- 

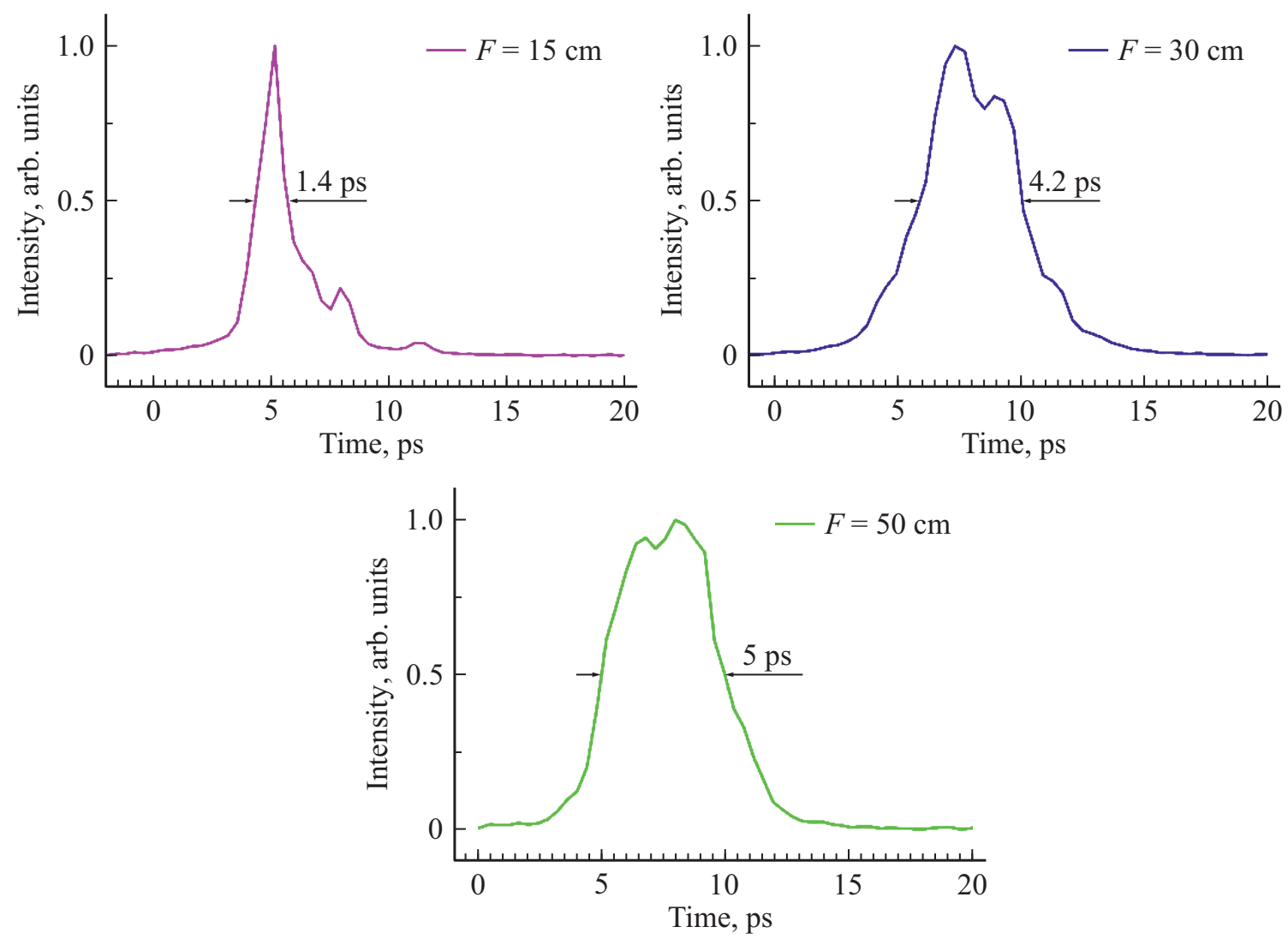

Рис. 5. Нормированные интенсивности импульса генерации во времени на длине волны 427.8 nm при фокусировке линзой с $F=15,30$ и $50 \mathrm{~cm}$. Нулевая точка соответствует времени прихода импульса накачки.

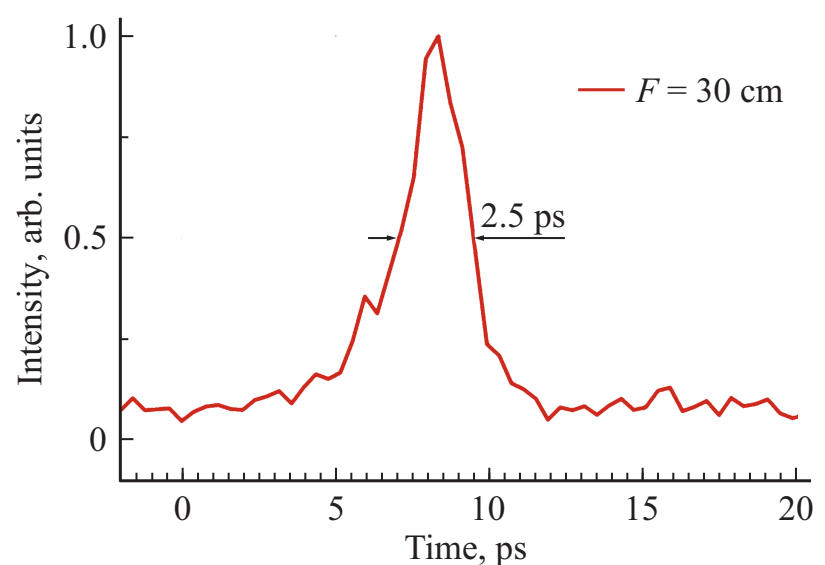

Рис. 6. Нормированная интенсивность импульса генерации во времени на длине волны $391.4 \mathrm{~nm}$ при фокусировке линзой c $F=30 \mathrm{~cm}$. Нулевая точка соответствует времени прихода импульса накачки.

лению $X(0)$. Вследствие этого произойдёт увеличение инверсии на переходе $B(0)-X(1)$ и её уменьшение на переходе $B(0)-X(0)$. В результате импульс генерации на длине волны $391.4 \mathrm{~nm}$ будет короче, чем на $427.8 \mathrm{~nm}$.

\section{Анализ лазерной плазмы и параметров активной среды}

Для того чтобы провести анализ параметров активной среды, важно знать геометрическое распределение и плотность активных частиц. Фотографии плазменных каналов при различной длине фокусировки представлены на рис. 7.

Продольные размеры наиболее яркой зоны плазменных каналов составляют 7, 10 и $12 \mathrm{~mm}$ для фокусных расстояний $F=30,40$ и $50 \mathrm{~cm}$ соответственно. Для $F=15 \mathrm{~cm}$ длина плазменного канала составляет $3 \mathrm{~mm}$ [7]. Средний диаметр плазмы - $(0.2-0.3) \mathrm{mm}$. Время прохода импульса излучения через такие плазменные каналы составляет 23,30 и 33 рs для $F=30,40$ и $50 \mathrm{~cm}$ соответственно.

Зная размеры активной среды (плазмы) и удельную мощность накачки, можно оценить концентрацию молекулярных ионов азота. Расчёты приведены для случая с $F=30 \mathrm{~cm}$ (размеры активной среды $7 \times 0.2 \mathrm{~mm}$, рис. 7,a). Удельная мощность $7.27 \cdot 10^{14} \mathrm{~W} / \mathrm{cm}^{3}$. Электронная плотность для такой удельной мощности излучения составляет $N_{e} \sim 5 \cdot 10^{17} \mathrm{~cm}^{-3}$ [19], т. е. начальная концентрация молекулярных ионов азота в лазерной плазме составляет $N_{i} \sim 5 \cdot 10^{17} \mathrm{~cm}^{-3}$. С учётом потерь в активной среде возможно получить $\sim 10^{17} \mathrm{~cm}^{-3}$ фото- 


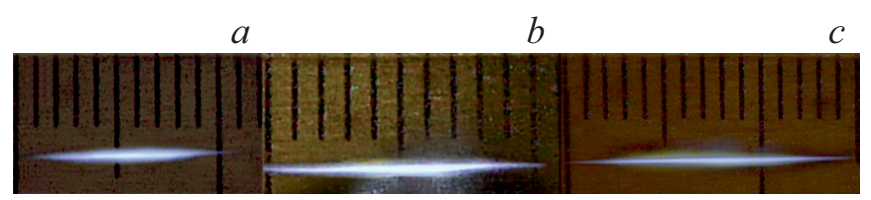

Рис. 7. Фотографии профилей плазменных каналов при фокусировке линзой с $F=30(a), 40(b)$ и $50 \mathrm{~cm}(c)$. Излучение распространяется справа налево. Одно деление соответствует $1 \mathrm{~mm}$.

нов или меньше из вынужденного излучения. Умножив на объём активной среды $\left(0.22 \cdot 10^{-3} \mathrm{~cm}^{3}\right)$, получим $2.2 \cdot 10^{13}$ фотонов из генерации. Это верхняя оценка инверсной населённости. Измеренная нами энергия генерации составляет $1-3 \mu \mathrm{J}$ при значении входной мощности накачки $0.16 \mathrm{TW}$. Полученное значение энергии хорошо согласуется с данными, полученными в работе [20]. Зная энергию генерации, можно получить реальное количество фотонов в импульсе. Это даёт $N_{\mathrm{ph}}=(2.1-6.5) \cdot 10^{12}$ фотонов. Уменьшение количества фотонов в реальных экспериментах можно объяснить высоким уровнем тушения верхнего лазерного уровня электронами плазмы, потерями на поглощение и рассеяние, конечным телесным углом усиливающей среды и возможностью заселения других состояний иона азота (например, $A^{2} \Pi_{u}$ ).

Одним из важных параметров активной среды является ненасыщенный коэффициент усиления $g_{0}$, который также можно оценить [8]. Известно, что увеличение интенсивности излучения во время усиления описывается как $I=I_{0} \exp \left(g_{0} L\right)$, где $I_{0}-$ начальная интенсивность, $L$ - длина активной среды. Следовательно, можно допустить, что генерация происходит при $g_{0} \geq 1 / L$. Если мы определим $L$ из длительности $\tau_{m}$, за которое фотонная лавина достигает своего максимума (рис. 5), тогда получим $L=0.15,0.21$ и $0.24 \mathrm{~cm}$ для $F=15$, 30 и $50 \mathrm{~cm}$ соответственно. Оценки коэффициента усиления для таких сред $g_{0} \geq 6.7,4.8$ и $4.2 \mathrm{~cm}^{-1}$. Полученные значения хорошо коррелируют с литературными данными, согласно которым коэффициент усиления $g_{0}$ на переходе $B^{2} \Sigma_{u}^{+}-X^{2} \Sigma_{g}^{+}$ионов молекулярного азота находится в диапазоне $\sim 1-6.7 \mathrm{~cm}^{-1}[1,3,7,12,21,22]$. Зная значение $g_{0}$, можно также оценить инверсную населённость активной среды на кубический сантиметр из выражения $\Delta N=g_{0} / \sigma$, где $\sigma$ - сечение индуцированного излучения. Например, для $g_{0} \geq 4.8 \mathrm{~cm}^{-1}$ и $\sigma=10^{-15} \mathrm{~cm}^{2}$ [23] получим $\Delta N \geq 1.05 \cdot 10^{12}$ фотонов. Полученное значение населённости хорошо согласуется с экспериментальным количеством фотонов $\left(2.1 \cdot 10^{12}\right)$ в импульсе генерации.

Как уже отмечалось выше, до настоящего времени открытым остаётся вопрос о пикосекундной длительности импульса генерации (1.15-7.8 ps) при усилении затравочного импульса с фемтосекундной длительностью (40-60 fs). Пока не ясно, почему так происходит и какие физические процессы за это отвечают. Мы предлагаем наше объяснение пикосекундной длительности генерации и её изменения при различных уровнях накачки. Фемтосекундный импульс затравки (гармоника, СК) имеет широкий спектр, который перекрывает область генерации. Во время распространения затравочных фотонов по активной среде часть из них, чья энергия совпадает с соответствующей энергией фотонов генерации, формирует импульс с длительностью, которая определяется спектральной шириной усиливаемого излучения. Минимальная длительность данного импульса может быть оценена из принципа неопределённости Гейзенберга как обратно пропорциональная соответствующей спектральной ширине. Порог генерации для различных частот зависит от уровня накачки: чем выше удельная мощность импульса накачки, тем больше коэффициент усиления и диапазон частот, на которых достигается порог генерации. Таким образом, изменяя уровень накачки, можно изменять ширину спектра, с которого начинается усиление, и тем самым задавать разную длительность импульса генерации. По мере усиления в активной среде спектрально ограниченного импульса к первоначальным частотам могут добавиться дополнительные, которые приведут к уширению спектра выходного излучения, но они уже не влияют на длительность импульса, сформированного при зарождении генерации, поскольку они не являются сфазированными за счет неоднородности и нестационарности плазмы.

\section{Заключение}

Исследованы параметры вынужденного излучения на переходе $B^{2} \Sigma_{u}^{+}-X^{2} \Sigma_{g}^{+}$иона молекулярного азота в воздушной лазерной плазме. Показано, что изменение параметров плазмы за счёт различных условий фокусировки пучка накачки позволяет изменять длительность импульса генерации от 1.15 до $4.6 \mathrm{ps}$, а ненасыщенный коэффициент усиления с 6.7 до $4.2 \mathrm{~cm}^{-1}$ соответственно. В одних и тех же экспериментальных условиях длительность импульса на длине волны $391.4 \mathrm{~nm}$ (2.1 ps) короче, чем длительность импульса на длине волны $427.8 \mathrm{~nm}$ (3.7 ps), что может быть связано с релаксацией колебательных уровней $1 \rightarrow 0$ основного электронного состояния $X^{2} \Sigma_{g}^{+}$. Предложен механизм формирования пикосекундной длительности импульса генерации, согласно которому импульс формируется в самом начале активной среды с длительностью, определяемой набором частот, на которых достигается порог генерации. При его усилении в активной среде набор частот может увеличиваться за счет столкновительного уширения электронами и спектр обычно расширяется вплоть до $\sim 0.3 \mathrm{~nm}$. Однако при этом сохраняется первоначальная длительность импульса. В связи с этим минимальная длительность импульса генерации (1.15 ps) в наших экспериментах была получена в активной среде с коэффициентом усиления $6.7 \mathrm{~cm}^{-1}$, когда порог генерации достигается на наибольшем количестве частот. 


\section{Финансирование работы}

Исследование выполнено при финансовой поддержке гранта РФФИ № 20-08-00060, гранта РФФИ и Томской области № 19-48-700016 и гос. задания FWRM-20210014.

\section{Конфликт интересов}

Авторы заявляют, что у них нет конфликта интересов.

\section{Список литературы}

[1] J. Yao, B. Zeng, H. Xu, G. Li, W. Chu, J. Ni, H. Zhang, S.L. Chin, Y. Cheng, Z. Xu. Phys. Rev. A, 84, 051802 (2011). DOI: 10.1103/PhysRevA.84.051802

[2] Y. Liu, Y. Brelet, G. Point, A. Houard, A. Mysyrowicz. Opt. Express, 21, 22791 (2013). DOI: 10.1364/OE.21.022791

[3] T.J. Wang, J.F. Daigle, S. Yuan, R. Li, S.L. Chin. Phys. Rev. A, 88, 053429 (2013). DOI: 10.1103/PhysRevA.88.053429

[4] J. Ni, W. Chu, C. Jing, H. Zhang, B. Zeng, J. Yao, G. Li, H. Xie, C. Zhang, H. Xu, S.L. Chin, Y. Cheng, Z. Xu. Opt. Express, 21, 8746 (2013). DOI: 10.1364/OE.21.008746

[5] D. Kartashov, J. Möhring, G. Andriukaitis, A. Pugžlys, A. Zheltikov, M. Motzkus, A. Baltuška. In: Proceedings of the CLEO, (SAN Jose, CA, USA, 6-11 May 2012), p. QTh4E.6.

[6] Pavel Polynkin. Air Lasing (Springer International Publishing AG, 2018).

[7] N.G. Ivanov, I.A. Zyatikov, V.F. Losev, V.E. Prokop'ev. Opt. Commun., 456, 124573 (2020). DOI: $10.1016 /$ j.optcom.2019.124

[8] I.A. Zyatikov, V.F. Losev, D.M. Lubenko, E.A. Sandabkin. Opt. Lett., 45 (23), 6518 (2020). DOI: 10.1364/OL.403577

[9] A. Mysyrowicz, R. Danylo, A. Houard, V. Tikhonchuk, X. Zhang, Z. Fan, Q. Liang, S. Zhuang, L. Yuan, Y. Liu. APL Photon., 4, 110807 (2019). DOI: 10.1063/1.5116898

[10] X. Zhong, Z. Miao, L. Zhang, Q. Liang, M. Lei, H. Jiang, Y. Liu, Q. Gong, C. Wu. Phys. Rev. A, 96, 043422 (2017). DOI: 10.1103/PhysRevA.96.043422

[11] J. Yao, G. Li, C. Jing, B. Zeng, W. Chu. New J. Phys., 15, 023046 (2013). DOI: 10.1088/1367-2630/15/2/023046

[12] J. Yao, W. Chu, Z. Liu, J. Chen, B. Xu, Y. Cheng. Appl. Phys. B, 124 (73), 72 (2018). DOI: $10.1007 / \mathrm{s} 00340-018-6940-\mathrm{y}$

[13] G. Li, C. Jing, B. Zeng, H. Xie, J. Yao. Phys. Rev. A, 89, 033933 (2014). DOI: 10.1103/PhysRevA.89.033833

[14] J. Qiao, P.A. Jaanimagi, R. Boni, J. Bromage, E. Hill. Rev. Sci. Instr., 84, 073104 (2013). DOI: 10.1063/1.4811145

[15] W. Chu, G. Li, H. Xe, J. Ni, B. Yao, H. Zhang, C. Jing, H. Xu, Y. Cheng, Z. Xu. Laser Phys. Lett., 11, 015301 (2014). DOI: 10.1088/1612-2011/11/1/015301

[16] D. Zhou, X. Zhang, Q. Lu, Q. Liang, Y. Liu. COL, 18, 203201 (2020).

[17] X. Zhong, Z. Miao, L. Zhang, H. Jiang, Y. Liu, Q. Gong, C. Wu. Phys. Rev. A, 97, 033409 (2018). DOI: 10.1103/PhysRevA.97.033409

[18] H. Zhang, C. Jing, J. Yao, G. Li, B. Zeng, W. Chu, J. Ni, H. Xie, H. Xu, S.L. Chin, K. Yamanouchi, Y. Cheng, Z. Xu. Phys. Rev. X, 3, 041009 (2013).

DOI: 10.1103/PhysRevX.3.041009
[19] F. Theberge, W. Liu, P.T. Simard, A. Becker, S.L. Chin. Chin. Phys. Rev. E, 74, 036406 (2006). DOI: 10.1103/PhysRevE.74.036406

[20] G. Point, Y. Liu, Y. Brelet, S. Mitryukovskiy, P. Ding, A. Houard, A. Mysyrowicz. Opt. Lett., 39 (7), 1725 (2014). DOI: 10.1364/OL.39.001725

[21] Z. Fan, X. Zhang, Q. Lu, Y. Luo, Q. Liang, L. Yuan, Z. Zhang, Y. Liu. Photonics, 7, 99 (2020). DOI: $10.3390 /$ photonics 7040099

[22] K. Zhai, Z. Li, H. Xie, C. Jing, G. Li, B. Zeng, W. Chu, J. Ni, J. Yao, Y. Cheng. COL, 13 (5), 050201 (2015).

[23] V.A. Dolgikh, I.G. Rusoi, A. Yu, A.M. Soroka. Soviet J. Quant. Electron., 18 (7), 854 (1988). DOI: 10.1070/QE1988v018n07ABEH012308 